\title{
The interplanetary magnetic decrease automatic detection (IMDAD) code
}

\author{
F. L. Guarnieri ${ }^{1}$, B. T. Tsurutani ${ }^{2}$, and E. Echer ${ }^{3}$ \\ ${ }^{1}$ Universidade do Vale do Paraiba (UNIVAP), Sao José dos Campos, SP, Brazil \\ ${ }^{2}$ Jet Propulsion Laboratory, California Institute of Technology, Pasadena, CA \\ ${ }^{3}$ Instituto Nacional de Pesquisas Espaciais, INPE, Sao Jose dos Campos, SP, Brazil
}

(Received February 7, 2008; Revised April 15, 2008; Accepted May 5, 2008; Online published May 29, 2009)

\begin{abstract}
A new code, called the interplanetary magnetic decrease (MD) automatic detection (IMDAD) code, has been developed to enable researchers in the field to rapidly identify MD events for further analyses. The criterion used for MD selection is $B_{\min }<X B_{0}$, where $X$ is a variable value and $B_{0}$ is the ambient magnetic field magnitude. The code can be applied to data sets from different instruments/missions located in different space plasma environments in the heliosphere. The code has been tested during slow solar wind, fast solar wind and CIR intervals at $\sim 5 \mathrm{AU}$ (from November 28 to December 03, 1992). For this test, we used a sliding window with a width of 300 seconds applied to 1-second high-resolution magnetic field data. The events identified by the code have been confirmed by hand analyses. The routine was able to identify 57 of the 118 MDs identified by hand $(\sim 50 \%)$. The selection criteria for IMDAD and hand-analyses MDs were not exactly the same, accounting for the different rates of occurrence. What is particularly encouraging is that IMDAD did not falsely identify any events. The discrepancies between the two methods are discussed in the text. This code will be made available to the general public.
\end{abstract}

Key words: Interplanetary magnetic decreases, solar wind, Alfvén waves.

\section{Introduction}

Magnetic Decreases (MDs; Turner et al., 1977) have been defined as $\sim 50 \%$ decreases in the interplanetary magnetic field magnitude (Winterhalter et al., 1994; Fränz et al., 2000; Tsurutani et al., 2003). These structures have been called a variety of names (magnetic holes, holes, magnetic dips, magnetic cavities, magnetic bubbles, etc.) due to their discovery at different times and in different locations of the heliosphere/Earth's magnetosphere (Sugiura et al., 1969; Turner et al., 1977; Luhr and Klocher, 1987; Winterhalter et al., 1994; Fränz et al., 2000). For events that have been examined to date, the magnetic pressure decreases have been supplanted by plasma thermal pressure increases, so that the entire structure is generally in pressure balance (Winterhalter et al., 1995; Burlaga, 1995; Fränz et al., 2000).

Most past studies have focused on large decreases of the magnetic field magnitude, events where the decrease magnitudes are larger than 0.5 times the ambient field. It is clear that this is an arbitrary threshold. Similar structures generated by the same mechanism, but lesser in intensity, are also present in the interplanetary medium. To allow the rapid detection of MDs of arbitrary intensity, a computer code called Interplanetary Magnetic Decrease Automatic Detection (IMDAD) has been developed for the space research. This code allows variable magnetic field decreases, variable inter-MD spacing, and can be applied to variable data rates. The code can be used to identify MDs of arbitrary length

Copyright (c) The Society of Geomagnetism and Earth, Planetary and Space Sciences (SGEPSS); The Seismological Society of Japan; The Volcanological Society of Japan; The Geodetic Society of Japan; The Japanese Society for Planetary Sciences; TERRAPUB by adjusting its window size (here we discuss only MDs of length up to $300 s$ to compare results to previous works). Although previous workers have developed routines to automatically identify MDs (Winterhalter et al., 1994; Fränz et al., 2000), those codes did not have the special features of IMDAD. The codes are also not available to the general public.

\section{Method of Analyses}

A general routine to identify MDs using high time resolution magnetometer data has been developed. The aim of the development of the IMDAD computer code is to be able to correctly identify MDs present in the data. In this paper, the routine will be described in detail. Pitfalls will also be discussed.

We have used the Ulysses high-resolution magnetometer data as our test sample. The data used for this test is nominally one-second (the Ulysses sampling rate varies) resolution magnetic field vector data for the interval from November 25 (day 330) to December 3 (day 338), 1992. Ulysses had recently encountered Jupiter (February, 1992) and was starting its first south polar pass. Ulysses was at $\sim-20^{\circ}$ and at 5.1 AU from the sun during the selected test data interval. This 9-day interval contained a variety of solar wind types. There are $\sim 4$ days of quiet solar wind, $\sim 3$ days of Corotating Interaction Region (CIR) solar wind, and $\sim 1$ day of non-CIR (pure) high speed stream solar wind.

IMDAD was tested on the above data interval. The MDs were identified by hand analyses and they were also independently identified by computer analyses. The MDs identified by hand but missed by computer were carefully examined to understand the reasons why. 
The definition of a MD used in this test is a decrease of field magnitude of $50 \%$ of the ambient magnetic field strength $\left(0.5 B_{0}\right)$. This is the same general criterion used by Winterhalter et al. (1994). This criterion is kept so there will be continuity of methods used in the literature (however, we will latter show that even with this simple definition, slightly different selection methods may yield significantly different results). We also hope to intercompare our results with those of others. It should also be noted by the reader that different decrease levels can be easily implemented. This program can be used by other scientists for analyses of MDs in the heliosphere and magnetosphere. Interested persons should contact F. L. Guarnieri at guarnieri@univap.br.

\subsection{Details of the IMDAD routine}

A sliding window of 300 seconds was used to calculate the average field magnitude $\left(B_{0}\right)$ during the interval. All points that have values less than $0.5 B_{0}$ are identified. The window is shifted by 1 second and the process repeated. This analysis is done for the entire data set. After the data has been initially processed in this manner, the same data points identified by different window placements and adjacent potential MD candidates are deleted (keeping only the beginning and end points). Single isolated points are also deleted, assuming that they are spurious data errors.

The window size of 300 seconds and the minimum MD separation of 30 seconds were empirically selected for this data set. We caution the reader that he/she might have to change these parameters for applications to different plasma regions. It would also be important that they empirically hand check the accuracy of the code as done in this paper.

A schematic showing the basics of the routine is indicated in Fig. 1. A nine-point interval with a sliding window size of three points is illustrated (only as an example) to give the reader a general idea of how the routine works. The sliding windows are represented by brackets A (black), B (blue), C (red), etc. For bracket A, the first 3 points are examined. The magnetic field average of the first three points is calculated. Any points that have values less than $50 \%$ of the average of the field are identified. In this case no points meet this criterion. Next, the window is slid by one point in time to give a new analysis subinterval (bracket $B$ in the figure). The result of the subsequent calculation is that point 4 satisfies the MD criterion. A blue star is noted above the point (for visualization). For the subinterval bracket $\mathrm{C}$, the average magnetic field is low, so no points are identified. The same is true for bracket D. For bracket E, point 5 will be detected as an MD. A yellow star is indicated above this point. After the entire interval has been analyzed, there are 2 points identified, points 4 and 5 .

The program next considers single MD points. If there is only a single isolated MD point, this is discarded assuming that it is a spurious data spike. In the example shown above, two adjacent points were selected, so there are no such single point cases.

The next step of the routine is to consider cases where there are several adjacent points. Point 5 is adjacent to point 4 so the program considers the deletion of point 5 . Next point 6 is examined. Since it is not identified as an $\mathrm{MD}$, point 5 is retained and identified as the end of the MD. Thus, the program identifies point 4 as the beginning of the

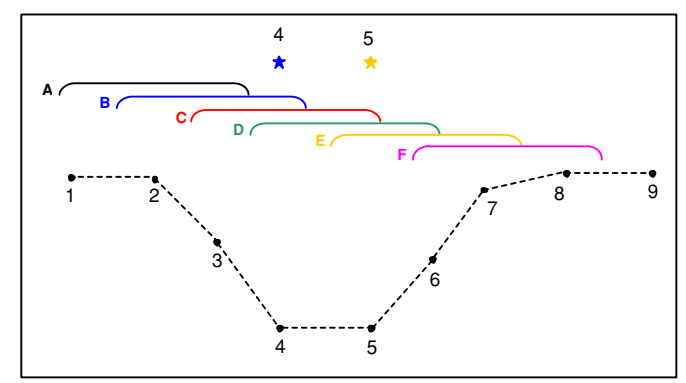

Fig. 1. Illustration of the general features of the routine. The stars over the brackets indicate the points that were identified as following the MDs criteria.

MD and point 5 as the end. This allows us to automatically determine the MD width.

The window width selected in our final analysis of the real Ulysses data was 300 seconds rather than 3 points, as shown in Fig. 1. The much shorter interval was chosen only to illustrate the general features of the program.

To be able to handle waves inside the MD or highly turbulent data, the program examines adjacent MDs that are closer than 30 seconds. Several different separation time scales, from 5 to 50 seconds were tested. The interval of 30 seconds was found to be the one with best correspondence to the hand-analyzed MD set, and it was selected for the present version of the program. If candidate MDs are closer together than 30 seconds, they are considered as a single MD. The two MDs are "merged" and the beginning of the first event and the end of the second become the boundaries of the new (merged) MD.

The MD duration indicated by the program is based only in the points that follow the MD criteria. The user would have to define what method he/she may find appropriate to identify the edges. Tsurutani et al. (1999), in their hand analyses of MDs, assumed an $1 / e$ decay from the $B_{0}$ calculated outside the MD. This is the criteria used in this paper in order to check the IMDAD code. Although we have stated the method used here, we state this as only an example. Other users can select their own criteria for edge detection.

\subsection{Hand analyses of MDs}

To test the accuracy of IMDAD MD selection, the same 9-day Ulysses magnetic field interval was examined by visual inspection (without reference to the computer results). For a potential MD, a background field on both sides of the MD was estimated by drawing line "averages" on a data plot. These were determined by visual inspection. Of the two "background fields", the higher value was used for the calculation. Next, the minimum field was determined by the same method. The $1 / e$ value of the difference between the highest background field and minimum field was required to be greater than $0.5 B_{0}$. This is the same method applied by Tsurutani et al. (1999).

\section{Results of the Intercomparison Between Computer-selected Events and Hand-selected Events}

Figure 2 shows the Ulysses data interval of the test. From top to bottom are the solar wind speed, proton density, 


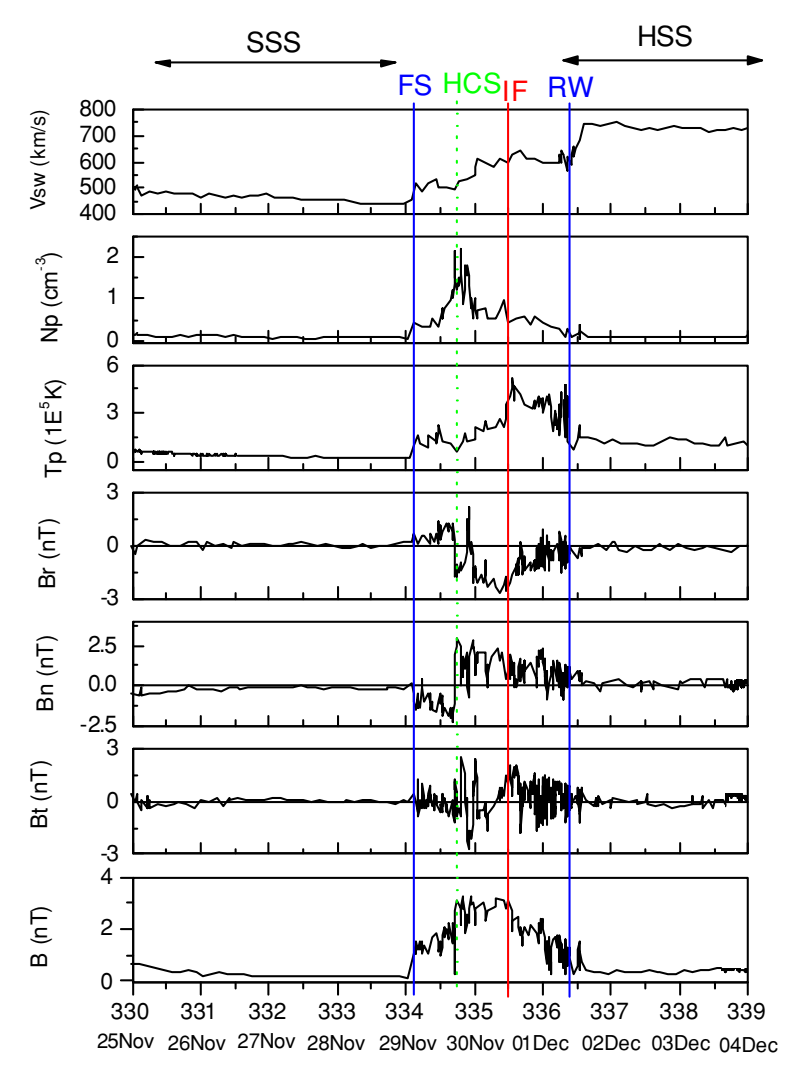

1992

Fig. 2. Ulysses data interval (November 25 to December 3, 1992) used to test the routine. The panels are, from top to bottom, the solar wind speed, proton density, proton temperature, magnetic field components, $R, T$ and $N$, and at the bottom, is the magnetic field magnitude. The slow speed stream is identified by SSS. The high speed stream is marked by HSS, and IF indicates the interface between the two regions. Present in the interval is a heliospheric current sheet, marked as HCS.

proton temperature, magnetic field components (in Solar Heliospheric $R, T$, and $N$ coordinates), and at the bottom, the magnetic field magnitude. At the top of the figure, the slow speed stream (SSS) and high speed stream (HSS) intervals are indicated by horizontal arrows. The slow speed stream is present from November 25 to 29 and the high speed stream from December 2 through 3. The CIR is the region of interaction of the two types of streams and is the interval between the slow and high speed streams proper. Some of the CIR structures are indicated for the interested reader. The CIR boundaries are a fast forward shock (FS, blue line) at the anti-solar direction boundary. A reverse wave (RW, blue line) is present at the solar direction boundary. The CIR occurs from 02:35 UT November 29 (day 334) to 11:30 UT December 1 (day 336). Several structures within the CIR are also indicated. There is an embedded heliospheric current sheet (HCS) indicated by a green line. The interface between the high speed stream and the slow speed stream is denoted by "IF" (for "interface"). The IF is indicated by a red line.

Figure 3 shows an example of a MD identified by the program. The panels are, from top to bottom, the magnetic field components $B_{R}, B_{T}$, and $B_{N}$, and the $B$ magnitude. This MD event, clearly visible in the $B$ magnitude panel, occurred on November 28, 1992 (DOY 333). The blue square

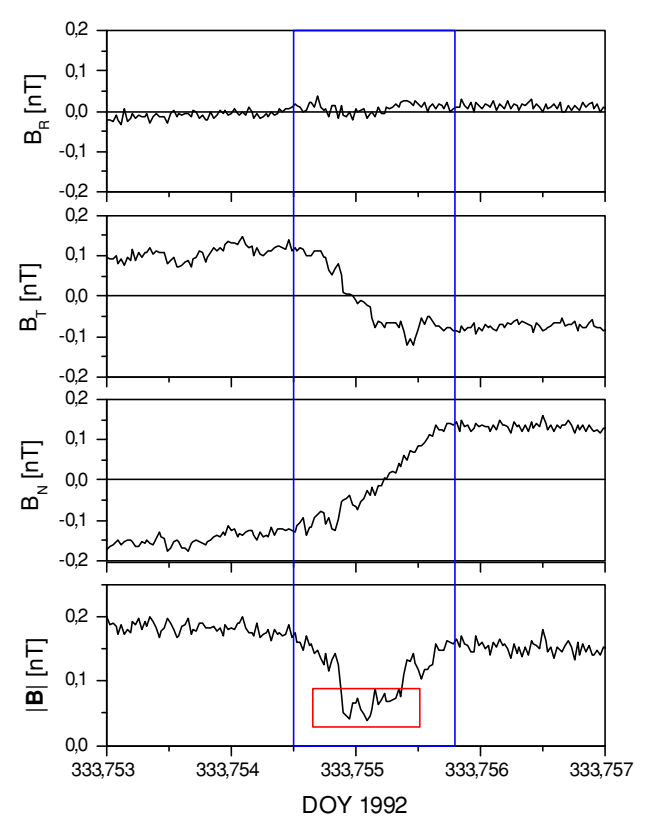

Fig. 3. Example of a MD identified by the program. The blue square marks the MD event, and the red box the MD identified by the program.

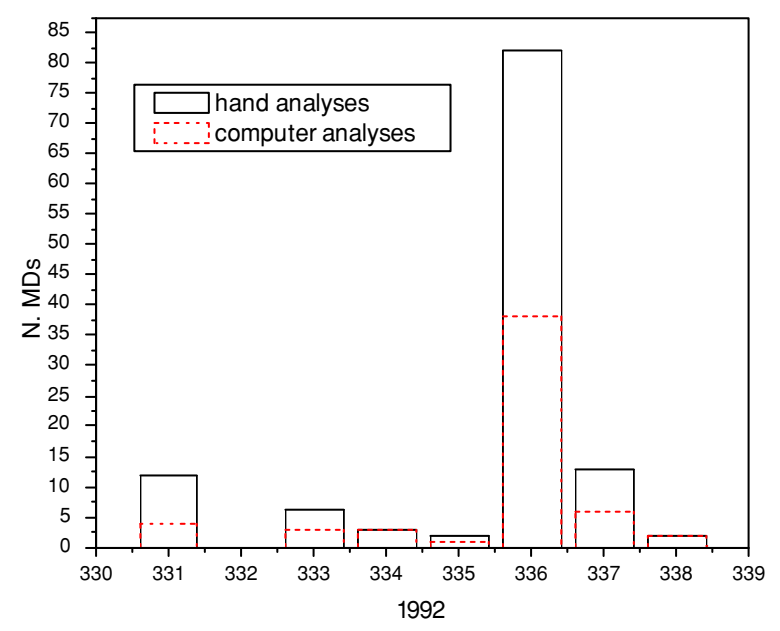

Fig. 4. MD occurrence rates using the two methods of analyses. The results from the computer analyses are plotted in dashed red and those from hand analyses are in solid black.

marks the MD event and the red box the MD identified by the program. There is a discontinuity in $B_{T}$ and $B_{N}$ components at the time of the MD occurrence. The $B_{T}$ component changes from $\sim 0.125 \mathrm{nT}$ to $\sim-0.1 \mathrm{nT}$ across the discontinuity. The change in $B_{N}$ is from $\sim-0.125 \mathrm{nT}$ to $\sim 0.15 \mathrm{nT}$.

The MD identified by the computer routine (red box) has a shorter duration when compared with hand analysis due to different identification procedures. In the IMDAD identification, the edges are based only in the points following the criteria, while in the hand analysis the inspectors used the borders values as the reference field to identify the MDs (the same method previously applied by Tsurutani et al., 1999).

The MD occurrence rates of the two methods of analyses are shown in Fig. 4. The top panel shows the number of MDs per day from the computer analyses in dashed red, 
and for those from hand analyses in solid black. The reader should note that the number of events from hand analyses is always greater or equal to the number from computer analyses. Over the whole interval, there are $118 \mathrm{MDs}$ identified by hand and 57 events identified by computer.

Each computer-selected event was compared with the events identified by hand. It is noted that all 57 computer selected events were also identified by hand analyses. This result gives confidence to the accuracy of the present program (and its internal parameters) used for this region of space, since no false positive MD was detected.

The causes for the code missing MDs were studied. It was found that the missed events fall into several categories. The two main causes were: 1) events where the magnetic field decrease is near the threshold of $-0.5 B_{0}$, and 2) when there are rapid oscillations in the magnetic field magnitude (if these oscillations have amplitudes comparable to the MD decrease, they can change the reference value $\left[B_{0}\right]$ for the window, while hand-analysis is not affected by these structures). In the first category there were 25 cases of "missed events" and in the second category there were 17 cases. Moreover, there are two minor causes of misidentification that we called "large MD" or "background contamination". The "large MD" refers to MDs that are longer than the window size used. These MDs can be selected by hand inspection but they may be missed by the computer since their durations are close to the window size. The "background contamination" usually occurs when there are discontinuities or shocks around the MD that may affect the $B_{0}$ calculation for the window.

For the first category of "missed events", the near "threshold" events, it is noted that the computer and hand analysis methods of determining the "background" field were slightly different. The hand analysis method used higher field values for the "background", so this criterion was less strict than the computer criterion. The computer method often contains field values in the decreasing slopes of the MDs, lowering the calculated "background" fields, giving stricter limits than hand analyses. Here the background field on both sides of the MD was estimated by drawing line "averages" on a data plot. Of the two "background fields", the higher value was used for the calculation (a more liberal assumption than the IMDAD code). Next, the minimum field was determined by the same method. The $1 / e$ value of the difference between the highest background field and minimum field was required to be greater than $0.5 B_{0}$. As the reader will readily note, although a simple condition of " $0.5 B_{0}$ " was used, this is a different criterion still. The point that we wish to emphasize is that slightly different criteria will yield a different set of events. Thus, the method of implementation of the criterion is important to understand. On the other hand, it is felt that no one method is better than another. All methods will give equally valuable information.

It should be obvious that the IMDAD "missed events" are not really "missed". Since hand-analyses and computer analyses will never have identical criteria, each is correct. It should be noted that the type of hand analyses done here and in Tsurutani and Ho (1999) is not exact and not 100\% reproducible.

\section{Summary and Discussion}

A computer program has been written which identifies heliospheric MDs in the Ulysses magnetic field data at $\sim 5 \mathrm{AU}$ from the sun. The program works well in the slow solar wind, in the pure high speed stream solar wind, and in the compression regions at the interface between slow and high speed streams (CIRs). The routine was able to identify 57 of the 118 events identified by hand analysis, about $50 \%$ of the events $(48.3 \%)$. However, all the 57 MDs identified by the program were also identified by hand analyses, giving confidence to the accuracy of the method. The events that were missed by the code were events that fell into two main categories: events that were slightly below the handtechnique threshold level of detection and events associated with "wave-like" intervals. For the first case, the slightly more liberal hand analyses method allowed the detection of these events. "Wave-like" events were generally found in hand analyses when the magnetic field magnitude was low. These events could possibly be compressional waves or small amplitude mirror mode (Tsurutani et al., 1982) structures. These low field magnitude regions are generally low plasma beta (the ratio of magnetic pressure to plasma thermal pressure) regions. Thus they could also be due to magnetic noise or turbulent structures.

Acknowledgments. Portions of this work were done at the Jet Propulsion Laboratory, California Institute of Technology, Pasadena CA under contract with NASA. EE would like to thank to the Brazilian FAPESP (2007/52533-1) and CNPq (PQ300104/2005-7 and 470706/2006-6) agencies for financial supports.

\section{References}

Burlaga, L. F., Interplanetary Magnetohydrodynamics, Oxford University Press, New York, 1995.

Fränz, M., D. Burgess, and T. S. Horbury, Magnetic depressions in the solar wind, J. Geophys. Res., 105, 12725, 2000.

Luhr, H. and N. Klocker, AMPTE-IRM observations of magnetic cavities near the magnetopause, Geophys. Res. Lett., 14, 186, 1987.

Sugiura, M., T. L. Skillman, B. Ledley, and J. P. Heppner, "Holes" in the magnetic field near the magnetopause, EOS Trans. AGU, 50, 278, 1969.

Tsurutani, B. T. and C. M. Ho, A review of discontinuities and Alfven waves in interplanetary space: Ulysses results, Rev. Geophys., 37, 517, 1999.

Tsurutani, B. T., E. J. Smith, R. R. Anderson, K. W. Ogilvie, J. D. Scudder, D. N. Baker, and S. J. Bame, Lion roars and non-oscillatory drift mirror waves in the magnetosheath, J. Geophys. Res., 87, 6060, 1982.

Tsurutani, B. T., G. S. Lakhina, D. Winterhalter, J. K. Arballo, C. Galvan, and R. Sakurai, Energetic particle cross-field diffusion: Interaction with magnetic decreases (MDs), Nonlin. Proc. Geophys., 6, 235, 1999.

Tsurutani, B. T., B. Dasgupta, J. K. Arballo, G. S. Lakhina, and J. S. Pickett, Magnetic field turbulence, electron heating, magnetic holes, proton cyclotron waves, and the onsets of bipolar pulse (electron hole) events: a possible unifying scenario, Nonl. Proc. Geophys., 21, 27, 2003.

Turner, J. M., L. F. Burlaga, N. F. Ness, and J. F. Lemaire, Magnetic holes in the solar wind, J. Geophys. Res, 82, 1921, 1977.

Winterhalter, D. H., M. Neugebauer, B. E. Goldstein, E. J. Smith, and S. J. Bame, and A. Balogh, Ulysses field and plasma observations of magnetic holes in the solar wind and their relation to mirror-mode structures, J. Geophys. Res., 99, 23372, 1994.

Winterhalter, D., M. Neugebauer, B. E. Goldstein, E. J. Smith, B. T. Tsurutani, S. J. Bame, and A. Balogh, Magnetic holes in the solar wind and their relation to mirror-mode structures, Space Sci. Rev., 72, 201, 1995.

F. L. Guarnieri (e-mail: guarnieri@univap.br), B. T. Tsurutani, and E. Echer 